\title{
Bilateral actigraphic quantification of upper extremity movement in hemiparetic children with perinatal stroke: a case control study
}

\author{
Asha Hollis ${ }^{1,2}$, Lauran Cole ${ }^{1,2}$, Ephrem Zewdie ${ }^{1,3}$, Megan J. Metzler ${ }^{4}$ and Adam Kirton ${ }^{1,3,5^{*}}$ (B)
}

\begin{abstract}
Background: Hemiparetic cerebral palsy impacts millions of people worldwide. Assessment of bilateral motor function in real life remains a major challenge. We evaluated quantification of upper extremity movement in hemiparetic children using bilateral actigraphy. We hypothesized that movement asymmetry correlates with standard motor outcome measures.

Methods: Hemiparetic and control participants wore bilateral wrist Actiwatch2 (Philips) for $48 \mathrm{~h}$ with movement counts recorded in 15-s intervals. The primary outcome was a novel statistic of movement asymmetry, the Actigraphic Movement Asymmetry Index (AMAI). Relationships between AMAl and standard motor outcomes (Assisting Hand Assessment, Melbourne Assessment, and Box and Block Test [BB]) were explored with Pearson or Spearman correlation.

Results: 30 stroke (mean 11 years 2 months ( 3 years 10 months); 13 female, 17 male) and 23 control (mean 11 years 1 month (4 years 5 months); 8 female, 15 male) were enrolled. Stroke participants demonstrated higher asymmetry. Correlations between AMAl and standard tests were moderate and strongest during sleep (BB: $r=0.68, p<0.01$ ).

Conclusions: Standard tests may not reflect the extent of movement asymmetry during daily life in hemiparetic children. Bilateral actigraphy may be a valuable complementary tool for measuring arm movement, potentially enabling improved evaluation of therapies with a focus on child participation.
\end{abstract}

Keywords: Hemiparetic cerebral palsy, Bilateral actigraphy, Real-life activity, Upper-extremity movement, Pediatrics

\section{Background}

Cerebral palsy (CP) accounts for most lifelong neurological disability and affects more than 17 million people worldwide [1-4]. Patients with hemiparetic cerebral palsy (HCP) suffer from motor dysfunction on one side of the body, often a result of acquired injury to the motor system, including the motor cortex or corticospinal tracts $[1,5]$. The leading cause of HCP is perinatal stroke due to a focal disruption in cerebral blood flow between

\footnotetext{
*Correspondence: adam.kirton@ahs.ca

${ }^{5}$ Pediatric Neurology, Alberta Children's Hospital, 28 Oki Drive NW, Calgary, AB T3B6A8, Canada

Full list of author information is available at the end of the article
}

20 weeks gestation and 28 days postpartum $[1,6,7]$. As a focal injury of defined timing in an otherwise healthy brain, perinatal stroke represents an ideal human model of developmental plasticity [8]. Since HCP is highly prevalent and treatment options are limited, new therapies are required to improve motor outcomes. Developmental preclinical and human models have facilitated clinical trials of non-invasive neuromodulation in hemiparetic children $[9,10]$. However, these and other promising trials could benefit from tools capable of measuring bilateral movement during real-life activities.

Validated tools to assess motor function in HCP are established. One example is the Assisting Hand Assessment (AHA), a bilateral measure of performance in original author(s) and the source, provide a link to the Creative Commons licence, and indicate if changes were made. The images or other third party material in this article are included in the article's Creative Commons licence, unless indicated otherwise in a credit line to the material. If material is not included in the article's Creative Commons licence and your intended use is not permitted by statutory regulation or exceeds the permitted use, you will need to obtain permission directly from the copyright holder. To view a copy of this licence, visit http://creativecommons.org/licenses/by/4.0/. The Creative Commons Public Domain Dedication waiver (http://creativeco mmons.org/publicdomain/zero/1.0/) applies to the data made available in this article, unless otherwise stated in a credit line to the data. 
which the affected arm may "assist" the unaffected arm in a series of predefined functional tasks [11]. As the AHA is designed to elicit bimanual interaction with objects, it may ultimately overestimate affected arm movement in day-to-day activities [12]. Another example is the Melbourne Assessment (MA), a unilateral upper extremity functional measure in which omission of bimanual function limits generalization to daily activity [13-15]. The Box and Block Test (BB) measures unilateral grasp and reach but offers limited insight into day-to-day function $[11,16,17]$. Although test-retest and inter-rater reliability are established, the AHA and MA are resource-intensive [16].

Such clinical measures are important for health providers to determine what functions and/or skills to target in therapy and to evaluate the success of therapy. However, they do not provide a full picture of function. Currently available measures suffer from limited ability to quantify upper extremity use in affected individuals in their natural environment. Aslam et al. point out that these standardized tests are validated, but reflect specific domains of function within the International Classification of Functioning, Disability, and Health (ICF) [18]. The ICF framework for functioning includes the domains of body structures, body functions, environment, and activity limitation in addition to highlighting interactions between domains. Standard clinical tests such as the AHA evaluate activity well, but do not provide an understanding of how activity limitations play out in an individual's real-life environment. In-situ assessment of unilateral and bilateral movement is potentially critical to a more complete picture of function for children with hemiparesis. One promising tool which may be able to address this need is actigraphy, a wearable technology. Actigraphs are lightweight, wrist-worn accelerometers that capture objective, detailed movement data in real time during normal community-based activities. When the watches accelerate, a voltage is produced which corresponds to the degree of acceleration, enabling actigraphy to reflect various intensities in movement $[7,19]$. Additional advantages include simplicity of use, and no lower age limit for application [15, 19-21]. As with other tools, actigraphy has challenges, including expensive devices, potential for missing data from participant noncompliance, and a lack of established guidelines for data analysis.

Actigraphy has seen limited use in children with HCP. Evidence supports the utility of actigraphic measures of motor function in normal children [19], adults with stroke [7], and some CP populations [20, 22-24], and suggests that bilateral actigraphy can measure real-world motor asymmetry in hemiparetic subjects [24-28]. Beani et al. collected bilateral actigraphy for typically-developing and hemiparetic children while they completed the AHA. Two main findings were reported: (1) they confirmed the validity of bilateral ActiGraph GT3X to measure upper limb motor asymmetry between typically-developing controls and children and youth with hemiparetic cerebral palsy; and (2) they demonstrated that an asymmetric activity count index (from actigraphic data) corresponds to impairment level in hemiparetic children [23]. Similarly, Hoyt et al. showed that accelerometers can detect asymmetries in upper extremity movements [28]. In further studies, they found moderate correlations between accelerometry metrics and the MA, demonstrating construct validity [24]. Actigraphy can only estimate movements and is therefore not a measure of function. However, in a child with hemiparesis, an ability to detect and quantify a relative change in movement of the impaired limb may be a useful proxy of spontaneous use and participation in normal life. Actigraphy thus represents a potentially valuable opportunity to better understand real-life outcomes in children with disabilities.

We aimed to evaluate the efficacy of bilateral actigraphy to quantify upper extremity movement in children with perinatal stroke and $\mathrm{HCP}$, hypothesizing that an actigraphic movement asymmetry index (AMAI) would positively correlate with standard clinical measures of upper extremity function. We also performed an exploratory analysis of the relationship between these standard measures and actigraphic data in relation to different levels of activity intensity.

\section{Methods \\ Populations}

Following approval by the Research Ethics Board of the University of Calgary (REB15-1742), two populations of participants were recruited for a case-control study from June 2016 to February 2017. Children with stroke aged 1 to 18 years with Magnetic Resonance Imagingconfirmed ischemic perinatal stroke (arterial or venous) were identified from a population-based research cohort (Alberta Perinatal Stroke Project) [29]. Additional criteria included unilateral stroke and no additional neurological conditions, severe developmental delays, or unstable epilepsy.

Typically developing participants aged 1 to 18 years were recruited from the Healthy Infants and Children Clinical Research Program (HICCUP, www.hiccupkids. ca). Typically developing participants had no neurological conditions or medications. Selection bias was minimized by recruiting comparable ages between stroke and control groups. All participants provided written informed consent/assent. 


\section{Actigraphy}

Following recruitment, participants were fitted with actiwatches (Actiwatch2, Philips Respironics, Pennsylvania) on both wrists at the Alberta Children's Hospital. Watches recorded movement data for a period of $48 \mathrm{~h}$ during routine life. Participants (or parents) were asked to record sleep and wake times on a standardized diary and press the event marker button at bedtime and waketime [30]. Motion data was captured in 15-s epochs. Actiware software (Philips Respironics) generated an Activity Count for each epoch. Times were manually excluded if the participant reported in the diary removal of the watches, such as bathing, as the watches were not waterproof. Rest intervals were generated from participants pressing the watch marker button at bedtime and wake-time and were validated using reported diary sleep and wake times. Sleep intervals were automatically determined by the Actiware sleep interval detection algorithm and could only occur within a rest interval.

Data was segmented according to intervals and/or activity levels. Intervals were segmented into [a] active, [b] rest, [c] sleep, and [d] all. Activity levels were defined for each 15-s epoch by the sum of the Activity Counts for both hands: [a] very low (total of $0-30)$, [b] low (31-160), [c] moderate (161-524), [d] high (525-812), [e] very high $(813+)$, and [f] all. Levels chosen matched those previously described [19] with the exception of two changes: an additional range of "very low" was added to expand analysis of low level movement, and all range limits were doubled to account for bimanual movement. Segmentation by both interval and activity level separated the data into 24 subgroups. It was therefore possible to have activity of different levels during all intervals including during sleep.

\section{Statistics}

A moment-by-moment asymmetry measure, which we label the Scaled activity difference (SAD), was calculated:

$$
\text { Scaled activity difference }(S A D)=\frac{L_{i t}-R_{i t}}{L_{i t}+R_{i t}}
$$

where $L_{i t}$ and $R_{i t}$ are the $\mathrm{AC}$ for the left and right hands in epoch of time $t$ for individual $i$. SAD was zero when there was no activity in either hand. Thus, SAD values ranged from -1 to 1 with -1 and 1 indicating only right and left arm movement, respectively, and values of 0 indicating equal left and right arm use. A SAD value was generated for each 15-s epoch for each participant. The SAD is similar to the "Asymmetry Index" used by Beani et al. and elsewhere, but assesses asymmetry at a given epoch, rather than as an average value [23]. For each individual, the SAD statistics were graphed to create a visual representation of the SAD score distribution for that participant, after being ranked from smallest to largest (Fig. 1).

We also constructed a novel summary statistic called the Actigraphic Movement Asymmetry Index (AMAI) as:

$$
A M A I=1-\left|(\operatorname{meanSAD})_{i j k}\right|
$$

for interval $j$ and level $k$ for individual $i$. The AMAI generated a single value to represent bilateral movement asymmetry in each participant for each interval $j$ and level $k$. Values range from 0 to 1 with closer to 1 indicating greater symmetry, a value of 1 indicating perfect symmetry, and a value of 0 indicating completely unilateral movement (fully asymmetric). A key feature of the AMAI is that it gives equal weight to all epochs regardless of intensity of upper limb activity. This accords value to both large and small movements, which are each important in everyday life. The AMAI was the primary outcome.

\section{Standard motor outcomes}

Standardized clinical motor outcomes, the AHA, MA and $\mathrm{BB}$, were obtained by experienced pediatric occupational therapists within the context of a clinical trial $[9,10]$. Measuring therapists were blinded to all patient details including stroke type, size and location at the time of assessment. Only the summary score for each test was used in the current analysis. Most BB scores were obtained during the trial measures; seven additional participants who were otherwise eligible but lacked recent AHA and MA scores received BB assessments from a trained research assistant. We also calculated the "Block Ratio" statistic, as described by Raglio [31] to provide a single value for $\mathrm{BB}$ scores and enable comparison with the AMAI:

$$
\text { Block Ratio }=\frac{\text { \# of blocks moved in } 1 \text { min by affected hand }}{\text { \# of blocks moved in } 1 \text { min by unaffected hand }}
$$

\section{Statistical analysis}

Associations were tested using Pearson correlation if data was normally distributed (Shapiro-Wilk test) and Spearman correlation if it was not. First, we examined correlations between different AMAI (actigraphy) intervals and levels. Second, we examined correlations between standard outcomes (AHA, MA, BB). Third, we tested for correlations between AMAI and the $\mathrm{AHA}, \mathrm{MA}$, and $\mathrm{BB}$ for all combinations of activity intervals (active, rest, sleep, all) and levels (very low, low, moderate, high, very high, all) for stroke participants only. No adjustments were made for participants without AHA or MA scores. Scatterplots provided 


\section{A) Distribution of Scaled Activity Difference Values: Control}
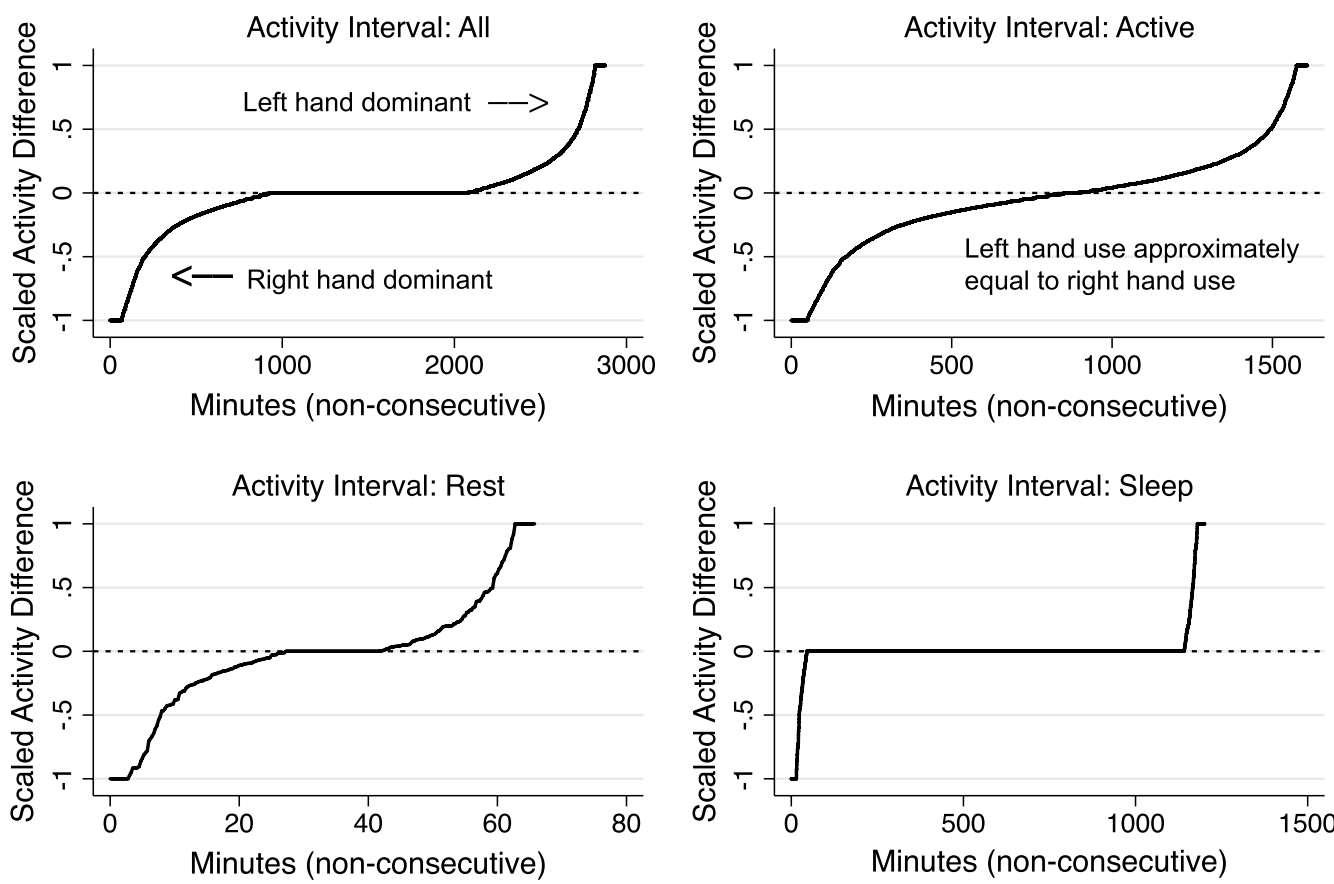

B) Distribution of Scaled Activity Difference Values: Stroke
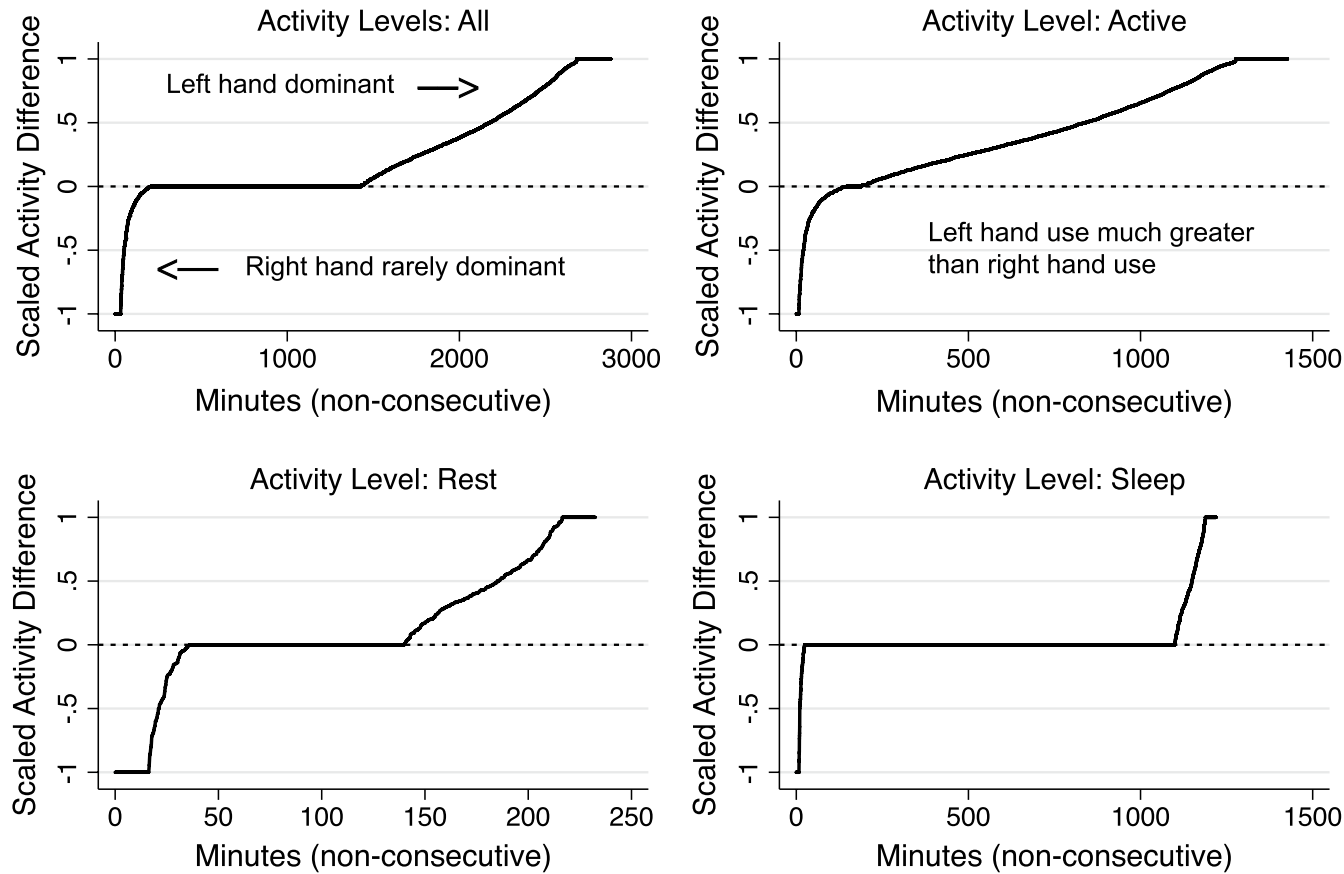

Fig. 1 Distribution of Scaled activity difference. All SAD values ranged from - 1 to 1 ; values of 1 indicate left arm use only, values of -1 indicate right arm use only, values of 0 indicate equal left and right arm use, values between 0 and 1 indicate left arm use is dominant, and values between 0 and -1 indicate right arm use is dominant. A Typically-developing participant: Note the high degree of rotational symmetry in all graphs, indicating nearly equal use of left and right arms. B Participant with stroke: Movement is markedly asymmetric with a low degree of rotational symmetry, indicating that left arm use is much greater than right arm use 
visual representations of selected relationships between variables.

Box plots of the AMAI compared differences between groups across activity intervals. Welch's $t$-test (unequal variances $t$-test) and Mann-Whitney $\mathrm{U}$ tests were applied depending on normality to compare groups. The tests were performed to systematically compare AMAI between participants with stroke and typicallydeveloping participants. As our primary outcome (AMAI) has not been previously described, a formal power calculation was not possible. However, based on similar approaches in the literature, a minimum sample of 25 stroke participants was estimated. Analysis was performed using Stata (version 14.2).

\section{Results}

Populations

53 individuals participated. Group characteristics, demographics and motor outcomes are summarized in Table 1. 30 stroke participants had a mean age of 11 years 2 months (SD 3 years 10 months; range 3 years 10 months -17 years 10 months; 13 female, 17 male). 23 typically-developing participants had a mean age of 11 years 1 month (SD 4 years 5 months; range 1 year 4 months-17 years 11 months; 8 female, 15 male). Groups did not differ significantly in age $(p=0.97)$ or $\operatorname{sex}(p=0.53)$. The AMAI was calculated for all participants. All three motor outcomes were obtained for 21 stroke participants while 7 had BB scores only. There were no adverse events and no drop-outs, and all stroke and control participants fully or partially filled out the diary.

\section{Actigraphy data}

The majority of epochs ( $57.7 \%$ stroke; $60.9 \%$ control) were distributed in active intervals between low and moderate activity levels. A large proportion of epochs (38.6\% stroke; $36.3 \%$ control) occurred in sleep intervals with very low activity levels. Within sleep, epochs of low to moderate activity levels were also present, but decreased in frequency as activity level increased. The overall distribution of epochs was comparable across both groups (Additional file 1: Table B). Participants wore the watches for an average of $96.7 \%$ of the time, with excluded time allowing for bathing or other activities involving water.

\section{Scaled activity difference (SAD)}

Scaled activity difference plots visually represent asymmetry of upper extremity movement across participants (Fig. 1). Patterns of asymmetry can be seen in SAD curves across all intervals. Movement curves among participants with stroke were characterized by rotational asymmetry. Comparison of SAD plots between participants with stroke and typically-developing participants visually represented the degree and nature of movement asymmetry among participants with stroke.

\section{Actigraphic movement asymmetry index (AMAl)}

AMAI scores spanned most of the possible range (from 0.03 to 0.99 out of 1 ) indicating that participants ranged from very asymmetric to highly symmetric. AMAI scores were widely distributed for participants with stroke but not for typically-developing participants, whose scores clustered near 1. Comparison of mean AMAI scores for stroke and typically-developing groups revealed statistically significant differences; the typically-developing

Table 1 Participant recruitment and characteristics

\begin{tabular}{|c|c|c|}
\hline & Participants with stroke & Typically-developing participants \\
\hline Eligible $(n)$ & 55 & 109 \\
\hline Approached $(n)$ & 44 & 37 \\
\hline Enrolled $(n)$ & 30 & 23 \\
\hline Actigraphy data $(n)$ & 30 & 23 \\
\hline Full set of AHA, MA and BB Scores (n) & 21 & $\mathrm{~N} / \mathrm{A}$ \\
\hline BB scores only (No AHA or MA) (n) & 7 & $\mathrm{~N} / \mathrm{A}$ \\
\hline Participants with BB scores $(n)$ & 28 & $\mathrm{~N} / \mathrm{A}$ \\
\hline Diary partially/fully completed $(n)$ & 30 & 23 \\
\hline $\operatorname{Sex}(n)$ & Male (17), Female (13) & Male (15), Female (8) \\
\hline Age range & 3 years 10 months -17 years 10 months & 1 year 4 months- 17 years 11 months \\
\hline Median age & 11 years 6 months & 12 years 2 months \\
\hline Mean age (SD) & 11 years 2 months (3 years 10 months) & 11 years 1 month (4 years 5 months) \\
\hline
\end{tabular}

Actigraphy data were collected from all stroke and control participants. Standard test scores were only available for the stroke cohort, and BB scores were either collected or available for $28 / 30$ participants. 21 of those participants also had AHA and MA scores. The control cohort had a slightly wider age range than the stroke cohort, although the median and mean ages were similar. There were more males than females in both cohorts 
group had higher symmetry across activity levels (Fig. 2, Additional file 1: Table A). The lone exception was high and very high levels during rest/sleep; however, these sections contained very limited data, typically $<0.1 \%$ of all data collected. AMAI scores correlated across activity levels with each other. Correlations were strongest

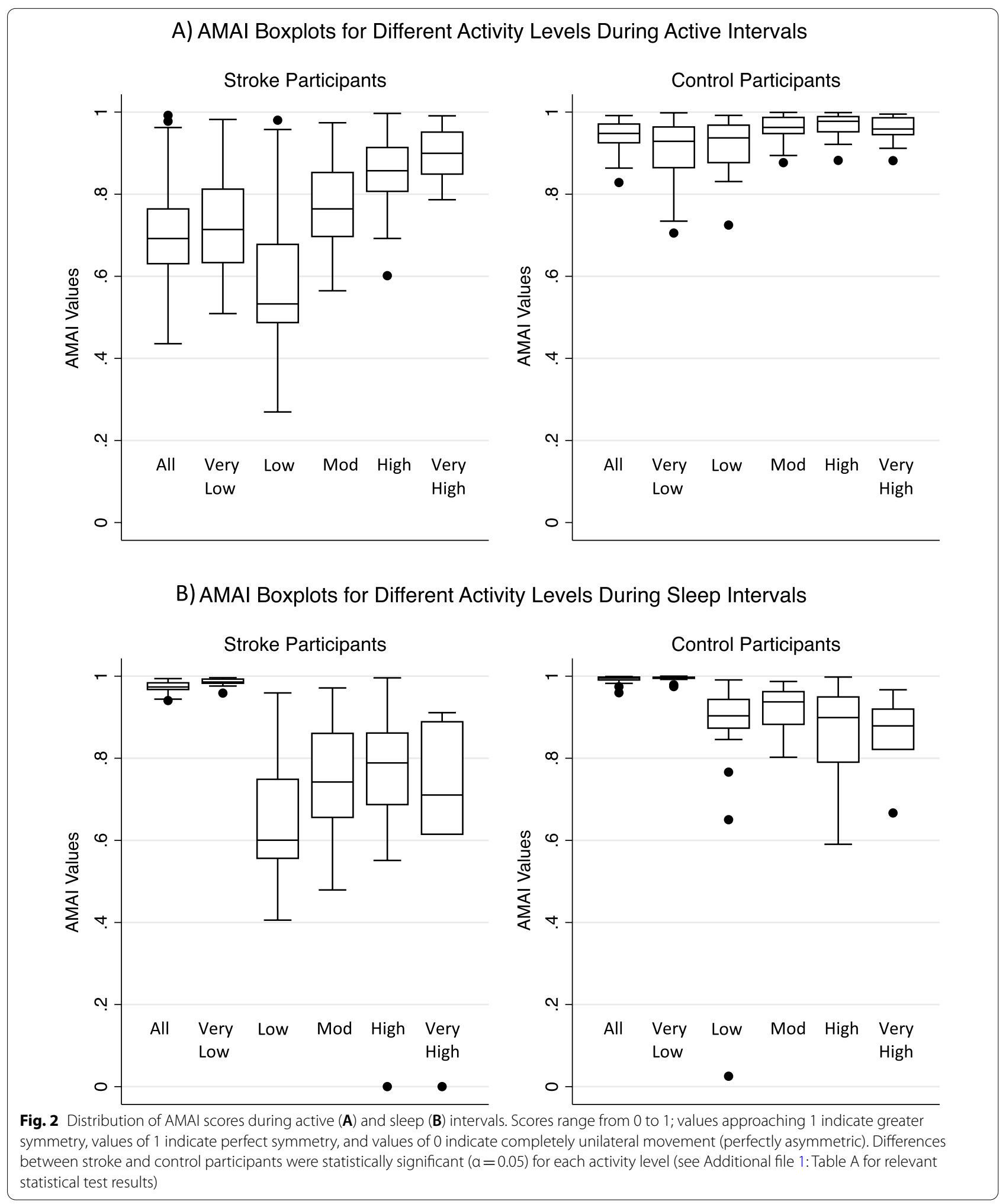


between adjacent activity levels while strength of correlation decreased the further the activity levels diverged (Fig. 3, Additional file 1: Table C).

\section{AMAl versus standard motor outcomes}

Modest correlations between standard tests and AMAI values were consistently observed. All motor outcomes correlated with the AMAI, but the highest associations were observed with the Block Ratio. Associations were strongest during sleep and rest intervals, and weaker but still significant during active intervals. Within sleep intervals, the strongest correlations were present in low and moderate activity levels (e.g. sleep, low activity, $r=0.68$, $p<0.01)$. Correlations were weaker for active intervals with very low or low activity levels (e.g. $r=0.42, p=0.03$ ) and not significant for very high activity levels (e.g. $r=0.21, p=0.28$ ) (Table 2). Strong correlations during sleep intervals remained after stratification by age. Low or moderate activity levels were most consistently correlated with the three motor outcomes, which also correlated strongly with each other. The strongest correlations were observed between the Block Ratio and the AHA and MA ( $r=0.93$ and 0.87 respectively). Correlation between the AHA and MA was also significant $(r=0.82)$.

\section{Discussion}

Our prospective cohort study suggests that bilateral wrist-worn actigraphs can measure asymmetry of upper limb movements in hemiparetic children during everyday life activities. Both the SAD graph and AMAI quantify characteristics of upper limb movements across activity levels. Correlations of varying strength between actigraphic data and standard assessments (BB, MA, AHA) suggests that standard assessments may not fully reflect real-life movement asymmetry. Actigraphy appears to be a practical way of capturing upper extremity movement both within clinical trials and normal life.

Bilateral actigraphy may offer some advantages for measuring upper extremity function in hemiparetic children. Actigraphy is not contaminated by learning and practice effects that may occur with repeated administration of standard measures. Actigraphy appears to represent a wide range of movement with continuous, objective measurement of movement asymmetry. Application before and after therapeutic interventions or longitudinally during development may be particularly useful. Varying strength of correlation between standard functional tests may indicate that standard tests do not fully reflect movement in

Relationship Between AMAI Scores for Different Levels During Active Intervals
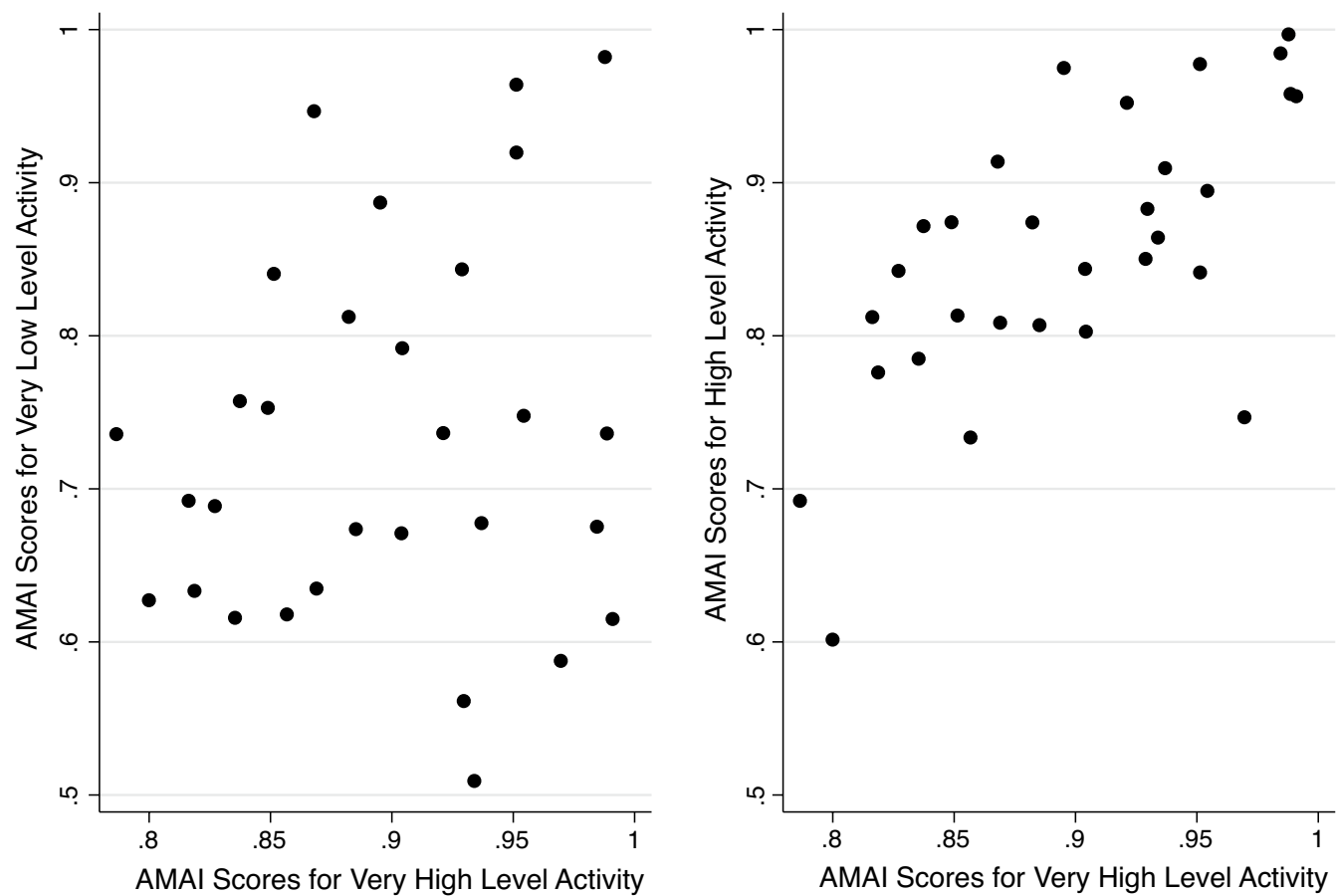

Fig. 3 Selected relationships for AMAI scores in active intervals. The scatter plot on the left represents the relationship between the AMAI "very low" and "very high" levels; the scatter plot on the right represents the relationship between the AMAl "high" and "very high" levels. Only the graph on the right shows a strong relationship, consistent with Additional file 1: Table C 
Table 2 Master correlation table

\begin{tabular}{|c|c|c|c|c|c|c|c|}
\hline \multirow[t]{2}{*}{ Interval } & \multirow[t]{2}{*}{ Level } & \multicolumn{2}{|l|}{ BB } & \multicolumn{2}{|l|}{ MA } & \multicolumn{2}{|l|}{ AHA } \\
\hline & & $r$ & p & $r$ & $p$ & $r$ & $p$ \\
\hline \multirow[t]{6}{*}{ Active } & Very low & 0.42 & 0.026 & 0.39 & 0.081 & $0.34^{\dagger}$ & 0.132 \\
\hline & Low & 0.49 & 0.008 & 0.4 & 0.07 & $0.36^{\dagger}$ & 0.112 \\
\hline & Moderate & 0.35 & 0.068 & 0.33 & 0.143 & $0.32^{\dagger}$ & 0.152 \\
\hline & High & 0.23 & 0.243 & 0.12 & 0.606 & $0.21^{+}$ & 0.36 \\
\hline & Very high & 0.21 & 0.284 & 0.06 & 0.792 & $0.08^{\dagger}$ & 0.741 \\
\hline & All & 0.42 & 0.027 & 0.39 & 0.076 & $0.40^{\dagger}$ & 0.071 \\
\hline \multirow[t]{6}{*}{ Sleep } & Very low & $0.17^{\dagger}$ & 0.45 & $0.10^{\dagger}$ & 0.68 & $0.12^{\dagger}$ & 0.607 \\
\hline & Low & 0.68 & $<0.001$ & 0.57 & 0.007 & $0.60^{\dagger}$ & 0.004 \\
\hline & Moderate & 0.56 & 0.003 & 0.48 & 0.027 & $0.50^{\dagger}$ & 0.021 \\
\hline & High & $0.49^{\dagger}$ & 0.086 & $0.42^{\dagger}$ & 0.149 & $0.44^{\dagger}$ & 0.135 \\
\hline & Very high & 0.12 & 0.883 & N/A & N/A & N/A & N/A \\
\hline & All & 0.43 & 0.026 & 0.41 & 0.066 & $0.48^{\dagger}$ & 0.027 \\
\hline \multirow[t]{6}{*}{ Rest } & Very low & $0.27^{\dagger}$ & 0.234 & $0.33^{\dagger}$ & 0.15 & $0.30^{+}$ & 0.191 \\
\hline & Low & 0.4 & 0.04 & 0.2 & 0.381 & $0.31^{\dagger}$ & 0.168 \\
\hline & Moderate & 0.57 & 0.002 & 0.69 & 0.001 & $0.74^{\dagger}$ & $<0.001$ \\
\hline & High & 0.26 & 0.215 & 0.28 & 0.259 & $0.20^{\dagger}$ & 0.434 \\
\hline & Very high & $0.67^{\dagger}$ & 0.05 & $0.67^{\dagger}$ & 0.05 & $0.40^{\dagger}$ & 0.284 \\
\hline & All & 0.35 & 0.071 & 0.35 & 0.118 & $0.50^{\dagger}$ & 0.02 \\
\hline \multirow[t]{6}{*}{ All } & Very low & $0.20^{\dagger}$ & 0.375 & $0.24^{\dagger}$ & 0.302 & $0.19^{\dagger}$ & 0.419 \\
\hline & Low & $0.42^{+}$ & 0.056 & $0.39^{\dagger}$ & 0.081 & $0.37^{\dagger}$ & 0.101 \\
\hline & Moderate & 0.36 & 0.058 & 0.35 & 0.12 & $0.32^{\dagger}$ & 0.151 \\
\hline & High & 0.24 & 0.216 & 0.14 & 0.546 & $0.24^{\dagger}$ & 0.293 \\
\hline & Very high & 0.21 & 0.273 & 0.07 & 0.769 & $0.10^{\dagger}$ & 0.657 \\
\hline & All & 0.46 & 0.014 & 0.35 & 0.117 & $0.33^{\dagger}$ & 0.15 \\
\hline
\end{tabular}

Pearson/Spearman Correlations between clinical motor outcomes and the AMAI. Correlations that were significant at the $a=0.05$ level are shown in bold text. Levels were defined for each 15-s epoch by the sum of the Activity Counts for both hands: [a] very low (total of 0-30), [b] low (31-160), [c] moderate (161-524), [d] high (525-812), [e] very high (813+), and [f] all

† Denotes Spearman's rho

everyday life. For example, correlations were strongest during sleep, a finding which may deserve further study. It is important to note that actigraphy metrics reflect different domains of functioning within the ICF. Specifically, actigraphy measures body functions (rather than activity) in real-world environments (rather than institutional environments). Thus actigraphy may be an important complement to standard tests. Whether actigraphy reflects what matters to individual children and families requires further study.

Consistent with existing literature in the field, our data are consistent with the feasibility of bilateral actigraphy in children: (1) no adverse effects were reported, (2) all participants were able wear the watches for many consecutive hours, and (3) all participants were able to fill out the diary tracking actiwatch use. Children, particularly those of school age, generally subjectively reported enjoying wearing the devices. Participant recruitment and enrolment were uncomplicated, and teaching families and arranging unit collection and return was not onerous.
Our study developed practical metrics to assess motor asymmetry during a wide variety of movement levels and types using actigraphy. The graphical display of SAD statistics appears to be a valuable way to transform raw actigraphy data into an easily interpreted visual representation of a wide range of asymmetries in bilateral upper extremity movement. Shifts in SAD curves may represent an informative new tool to examine the effects of interventions designed to increase real-world upper extremity use. The AMAI offers a single representative summary statistic per participant, enabling examination of movement within individuals across activity levels and between groups. The wide range of scores observed appears to reflect the expected spectrum of physical disability. Application across populations and within interventional trials will be required to determine the utility of the SAD graphs and AMAI. Additionally, another potential avenue of use may include motor function assessment or even early diagnosis of cerebral palsy in very young children. 
Important limitations are acknowledged. Actigraphy outcomes were only compared with available standard tests, which themselves may be imperfect. The fact that actigraphy data correlated with, but were different from, these established measures does not mean one is necessarily better than the other. As discussed earlier, these tools likely reflect different aspects of upper extremity function. Other limitations include our modest sample size which may have limited our ability to fully define the utility of actigraphic measurements. For some participants, there was a delay of several months between the collection of our standard test scores and actigraphy data which may have affected the correlations reported. Our actiwatches were also not designed to capture brief, smaller hand movements, which occur over a few seconds and were beyond the temporal resolution of our methods $[13,16]$. In addition, although actigraphy may capture movement asymmetry, further validation is needed to verify that activity counts fully reflect functional limb use: actigraphy may be biased by impaired movement patterns, resulting in an elevated movement count not attributable to functional use.

\section{Conclusions}

Overall, three main findings emerged from this research. First, actigraphy appears to be able to measure dailylife motor asymmetry in both typically-developing and hemiparetic children. Second, using the SAD graphs and AMAI statistics, actigraphy can assess the range of everyday bilateral upper-extremity motor asymmetries. Finally, the diversity in everyday movement of children with hemiparesis captured by actigraphy suggests that the standard tests may not fully represent movement asymmetries.

The translational value of detecting change in realworld asymmetry of upper limb movement in hemiparetic children may be game-changing. Modern models of pediatric rehabilitation have moved towards intensive treatment interventions over short time periods, often attempting to deliver high doses of therapy over weeks. While such approaches can yield measurable changes in function according to standard measures, effect sizes are often modest with only a portion of subjects "responding". However, more encouraging is evidence that small changes produced in the short-term grow over subsequent months and years. Our recent trial of constraint therapy and brain stimulation in hemiparetic children observed this phenomenon with sustained or increased gains at 6 months [9]. Even in the developed brain, the effects of 2 weeks of constraint therapy in adult stroke hemiparesis have been shown to increase at 2 years follow-up [32]. These results suggest that small increases in the use of a hemiparetic limb resulting from an acute intervention may amplify over time to realize more significant long-term functional benefits. If true, such effects are almost certainly more important in the developing brains of young children. Bilateral actigraphy brings new promise to better measure and understand such developmental effects of neurorehabilitation.

In addition to the replication of our findings, important future directions include the use of bilateral actigraphy in prospective, controlled clinical trials of pediatric hemiparesis. We are employing this approach in a current multicenter clinical trial of non-invasive brain stimulation combined with intensive therapy in hemiparetic children with perinatal stroke (NCT03216837). Such efforts to validate actigraphy hold promise in the development of real-life measures of rehabilitation interventions.

\section{Abbreviations \\ AHA: Assisting Hand Assessment; AMAl: Actigraphic Movement Asymmetry Index; BB: Box and Blocks Test; CP: Cerebral palsy; HCP: Hemiparetic cerebral palsy; HICCUP: Healthy Infants and Children Clinical Research Program; ICF: International Classification of Functioning, Disability, and Health; MA: Mel- bourne assessment; SAD: Scaled activity difference.}

\section{Supplementary Information}

The online version contains supplementary material available at https://doi. org/10.1186/s12984-021-00962-9.

Additional file 1: Table A. Comparing Mean AMAI Scores for stroke and control cohorts. Table B. Distribution of Epochs. Table C. Pearson correlations between different AMAI activity levels in active intervals for typically-developing participants.

\section{Acknowledgements}

Support from Amalia Floer, Jacquie Hodge and Helen Carlson in their generous assistance with participant recruitment and meetings is gratefully acknowledged. This study is supported by a Grant from CIHR (10167).

\section{Authors' contributions}

All listed authors meet the appropriate authorship criteria and no authors have been omitted. AH contributed to the study design, recruitment of participants, data acquisition and analysis, and drafting and revising the manuscript. EZ contributed to the study design, and drafting and revising the manuscript. LC and MM contributed to the data acquisition and analysis, and drafting the manuscript. AK contributed to obtaining funding, study design, data analysis, and drafting and revising the manuscript. All authors had access to study data. All authors read and approved the final manuscript.

\section{Funding}

This study was supported by Grant 10167 from CIHR.

\section{Availability of data and materials}

The datasets used and/or analysed for this study are available from the corresponding author upon reasonable request.

\section{Declarations}

Ethics approval and consent to participate

This study has been approved by the Research Ethics Board of the University of Calgary (REB15-1742). 


\section{Consent for publication}

Not applicable.

\section{Competing interests}

The authors declare that they have no competing interests.

\section{Author details}

${ }^{1}$ Pediatrics, Cumming School of Medicine, University of Calgary, Calgary, Canada. ${ }^{2}$ Neurosciences, Cumming School of Medicine, University of Calgary, Calgary, Canada. ${ }^{3}$ Medicine, Cumming School of Medicine, University of Calgary, Calgary, Canada. ${ }^{4}$ Clinical Neurosciences, Alberta Children's Hospital, Calgary, Canada. ${ }^{5}$ Pediatric Neurology, Alberta Children's Hospital, 28 Oki Drive NW, Calgary, AB T3B6A8, Canada.

Received: 25 September 2020 Accepted: 17 November 2021 Published online: 16 December 2021

\section{References}

1. Kirton A, deVeber G. Life after perinatal stroke. Stroke. 2013;44:3265-71.

2. Kirton A, DeVeber G. Paediatric stroke: pressing issues and promising directions. Lancet Neurol. 2015;14:92-102.

3. Oskoui M, Coutinho F, Dykeman J, Jetté N, Pringsheim T. An update on the prevalence of cerebral palsy: a systematic review and meta-analysis. Dev Med Child Neurol. 2013:55:509-19.

4. Dunbar M, Mineyko A, Hill M, Hodge J, Floer A, Kirton A. Population based birth prevalence of disease-specific perinatal stroke. Pediatrics. 2020:146:e2020013201.

5. Kulak W, Sobaniec W. Comparisons of right and left hemiparetic cerebral palsy. Pediatr Neurol. 2004;31:101-8.

6. Raju TNK, Nelson KB, Ferriero D, Lynch JK, NICHD-NINDS Perinatal Stroke Workshop Participants. Ischemic perinatal stroke: summary of a workshop sponsored by the National Institute of Child Health and Human Development and the National Institute of Neurological Disorders and Stroke. Pediatrics. 2007;120:609-16.

7. Seitz RJ, Hildebold T, Simeria K. Spontaneous arm movement activity assessed by accelerometry is a marker for early recovery after stroke. J Neurol. 2011;258:457-63.

8. Kirton A. Modeling developmental plasticity after perinatal stroke: defining central therapeutic targets in cerebral palsy. Pediatr Neurol. 2013:48:81-94.

9. Kirton A, Andersen J, Herrero M, Nettel-Aguirre A, Carsolio L, Damji O, et al. Brain stimulation and constraint for perinatal stroke hemiparesis: The PLASTIC CHAMPS Trial. Neurology. 2016;86:1659-67.

10. Kirton A, Ciechanski P, Zewdie E, Andersen J, Nettel-Aguirre A, Carlson H, et al. Transcranial direct current stimulation for children with perinatal stroke and hemiparesis. Neurology. 2017;88:259-67.

11. Krumlinde-sundholm L, Eliasson A-C. Development of the assisting hand assessment: a Rasch-built measure intended for children with unilateral upper limb impairments. Scand J Occup Ther. 2003;10:16-26.

12. Uswatte G, Taub E, Griffin A, Vogtle L, Rowe J, Barman J. The pediatric motor activity log-revised: assessing real-world arm use in children with cerebral palsy. Rehabil Psychol. 2012;57:149-58.

13. Johnson LM, Randall M, Reddihough D, Oke LE, Burt TA, Bach T. Development of a clinical assessment of quality of movement for unilateral upper-limb function. Dev Med Child Neurol. 1994;36:965-73.

14. The Melbourne Assessment 2: About the Melbourne Assessment 2. http://www.rch.org.au/melbourneassessment/. Accessed 2 Mar 2017.

15. Michielson ME, Selles RW, Stam HJ, Ribbers GM, Bussmann JB. Quantifying nonuse in chonric stroke patients: a study into paretic, nonparetic, and bimanual upper-limb use in daily life. Arch Phys Med Rehabil. 2012;93:1975-81.

16. Gilmore R, Sakzewski L, Boyd R. Upper limb activity measures for 5- to 16-year-old children with congenital hemiplegia: a systematic review. Dev Med Child Neurol. 2010;52:14-21.

17. Mathiowetz V, Volland G, Kashman N, Weber K. Adult norms for the Box and Block Test of manual dexterity. Am J Occup Ther. 1985:39:386-91.

18. Aslam R, van Bommel A, Southwood T, Hackett J, Jester A. An evaluation of paediatric hand and upper limb assessment tools within the framework of the World Health Organisation International Classification of Disability Functioning and Health. Hand Ther. 2015;20:24-34.

19. Ekblom O, Nyberg G, Bak E, Ekelund U, Marcus C. Validity and comparability of a wrist-worn accelerometer in children. J Phys Act Health. 2012;9:389-93.

20. Keawutan P, Bell KL, Oftedal S, Davies PSW, Boyd RN. Validation of accelerometer cut-points in children with cerebral palsy aged 4 to 5 years. Pediatr Phys Ther. 2016;28:427-34.

21. Sokal B, Uswatte G, Vogtle L, Byrom E, Barman J. Everyday movement and use of the arms: relationship in children with hemiparesis differs from adults. J Pediatr Rehabil Med. 2015;8:197-206.

22. Coker-Bolt P, Downey R, Connolly J, Hoover R, Shelton D, Seo N. Exploring the feasibility and use of acceleromters before, during, and after a campbased CIMT program for children with cerebral palsy. J Pediatr Rehabil Med. 2017;10:27-36.

23. Beani E, Maselli M, Sicola E, Perazza S, Cecchi F, Dario P, et al. Actigraph assessment for measuring upper limb activity in unilateral cerebral palsy. J NeuroEng Rehabil. 2019;16:30.

24. Hoyt CR, Brown SK, Sherman SK, Wood-Smith M, Van AN, Ortega M, et al. Using accelerometry for measurement of motor behavior in children: relationship of real-world movement to standardized evaluation. Res Dev Disabil. 2019;96:103546.

25. Bailey RR, Klaesner JW, Lang CE. Quantifying real-world upper-limb activity in nondisabled adults and adults with chronic stroke. Neurorehabil Neural Repair. 2015;29:969-78.

26. Hayward KS, Brauer SG. Dose of arm activity training during acute and subacute rehabilitation post stroke: a systematic review of the literature. Clin Rehabil. 2015;29:1234-43.

27. Noorkõiv M, Rodgers H, Price CL. Accelerometer measurement of upper extremity movement after stroke: a systematic review of clinical studies. J NeuroEng Rehabil. 2014;11:144.

28. Hoyt CR, Van AN, Ortega M, Koller JM, Everett EA, Nguyen AL, et al. Detection of pediatric upper extremity motor activity and deficits with accelerometry. JAMA Netw Open. 2019;2:e192970-e192970.

29. Kirton A, Deveber G, Pontigon A-M, Macgregor D, Shroff M. Presumed perinatal ischemic stroke: vascular classification predicts outcomes. Ann Neurol. 2008;63:436-43.

30. Martin JL, Hakim AD. Wrist actigraphy. Chest. 2011;139:1514-27.

31. Raglio A, Panigazzi M, Colombo R, Tramontano M, losa M, Mastrogiacomo $\mathrm{S}$, et al. Hand rehabilitation with sonification techniques in the subacute stage of stroke. Sci Rep. 2021;11:7237-7237.

32. Wolf SL, Winstein CJ, Miller JP, Thompson PA, Taub E, Uswatte G, et al. Retention of upper limb function in stroke survivors who have received constraint-induced movement therapy: the EXCITE randomised trial. Lancet Neurol. 2008;7:33-40.

\section{Publisher's Note}

Springer Nature remains neutral with regard to jurisdictional claims in published maps and institutional affiliations.

Ready to submit your research? Choose BMC and benefit from

- fast, convenient online submission

- thorough peer review by experienced researchers in your field

- rapid publication on acceptance

- support for research data, including large and complex data types

- gold Open Access which fosters wider collaboration and increased citations

- maximum visibility for your research: over 100M website views per year

At BMC, research is always in progress.

Learn more biomedcentral.com/submissions 